\title{
Role of GeneXpert in Rapid Molecular Detection of Extrapulmonary Tuberculosis in Tertiary Care Hospital
}

\author{
Yadhav K. ${ }^{1}$, Veena M. $^{2}$ \\ ${ }^{1}$ Dr. Kala Yadhav M L, Professor, ${ }^{2}$ Dr. Veena M, Post Graduate, both authors are attached with Department of \\ Microbiology, Bangalore Medical College and Research Institute, Karntaka, India.
}

Corresponding Author: Dr. Kala Yadhav M L, Professor, Department of Microbiology, Bangalore Medical College and Research Institute, Karntaka, India. E-mail: kalayadv@rediffmail.com

\begin{abstract}
Introduction: Tuberculosis, the most common infectious disease with prevalence of 9.6 million globally. Most prevalent $(23 \%)$ in India. Extrapulmonary tuberculosis (EPTB) accounts for $20 \%$ of total burden of tuberculosis. Rapid detection of Mycobacterium Tuberculosis (MTB) is essential for effective disease management. CBNAAT (Cartridge Based NucleicAcid Amplification Test) or GeneXpert MTB/RIF assay - novel diagnostic tool to detect MTB and RIF resistance simultaneously. WHO recommends its utility for non-respiratory samples also. Burden of EPTB and drug resistance vary from place to place. Objective: Study was conducted to gather information about burden of disease in our locality and to assesutility of CBNAAT in detecting MTB and rifampicin resistance in suspected EPTB cases. Methods: Retrospective analysis of 281 samples from suspected cases collected in falcon tubes and processed using CBNAAT. Result: Total of 281 extrapulmonary samples received, 67(23.8\%) were positive and 214(76.1\%) were negative for MTB. Of 67 positives, RIF resistance detected in 1(1.49\%) case. Maximum number of MTB detected in the age group 21-30 years (n=23, 34.3\%). Among 165 males and 116 females, MTB detected in 44(26.6\%) and 23(19.8\%) respectively. Out of 281 patients, 24(8.54\%) were HIV positive. Of these 24, only 8(33.3\%) found positive for MTB. Among 257 non-HIV patients, MTB detected in 59(22.9\%). Among different samples received, maximum number were Pleural fluid $\mathrm{n}=115(40.9 \%$ ) and Maximum MTB positives found in FNAC (of lymphnodes) samples [n=35(52.2\%)]. Conclusion: CBNAAT is a rapid test to detect MTB and rifampicin resistance simultaneously in EPTB and it reduced the treatment abuse in suspected cases.
\end{abstract}

Key words: CBNAAT, Extrapulmonary TB, MTB, rifampicin resistance.

\section{Introduction}

Tuberculosis (TB) is the most common infectious disease and in developing countries like India, it is major health problem. Due to inadequate diagnostic assays it remains as a challenge to public health. According to the WHO Tuberculosis report in 2015, there are 9.6 million people infected with TB. India has the maximum number of cases, that is $23 \%$ of the total cases all over the world. Globally, an estimated $3.3 \%$ of new TB cases and $20 \%$ of previously treated cases have MDR-TB [1]. Extra Pulmonary TB (EPTB) can affect any organ in the body and it accounts for $20 \%$ of total burden of tuberculosis globally. In majority of cases it remains undetected for a longer time. A major hindrance to the diagnosis of EPTB is the atypical

Manuscript received: $26^{\text {th }}$ May 2018

Reviewed: $4^{\text {th }}$ June 2018

Author Corrected: $10^{\text {th }}$ June 2018

Accepted for Publication: $15^{\text {th }}$ June 2018 presentation, often simulating neoplasia and/or inflammatory disorders and clinical specimens of deep organs are difficult to obtain. Adding to this, the Mycobacterium Tuberculosis Bacilli (MTB) load is generally very low in non-respiratory samples, therefore strongly affecting the sensitivity of acid-fast microscopy [2]. Also quick and reliable laboratory diagnostic methods for detecting tubercle bacilli in EPTB specimens are not easily available. This adds to the increased rates of morbidity and mortality in EPTB patients [3].

As the conventional laboratory methods are slow and cumbersome, Foundation for Innovative New Diagnostics (FIND) introduced cartridge-based nucleic acid amplification assay (GeneXpert MTB/rifampicin [RIF]). It is a molecular test which is fully automated and detects MTB directly from clinical samples within 


\section{Original Research Article}

two hours as well as RIF resistance which is the surrogate marker of MDR-TB conferring mutations in 81 bp RIF resistance determining region (RRDR) of the rpoB gene, which codes for a beta subunit of RNA polymerase of MTB, is the genetic basis of RIF resistance [4]. In 2014, The GeneXpert MTB/RIF assay has been strongly recommended by WHO for testing non-respiratory specimens from patients suspected of having EPTB to diagnose MTB and multidrug-resistant TB over the conventional tests (including microscopy, culture or histopathology). The test is currently recommended as a "first line" fast diagnostic test in high TB burden countries like India [5,6]. Burden of EPTB and drug resistance vary from place to place, therefore this study was conducted to assess the burden of EPTB and drug resistance in our locality.

\section{Materials and Methods}

Type and place of study: Study was a retrospective observational record-based analysis conducted in the RNTCP lab attached to the Department of Microbiology in Bowring and lady Curzon hospital.

Sample collection: Total of 281 clinically suspected EPTB cases were received in RNTCP lab from various department of our hospital and other private hospitals. Few details of the patients like Name, Address, Age,
Sex, HIV status, Treatment received and Name of the referring centers were noted down.

Study period: From May 2016 to April 2018.

Sampling method: Extra pulmonary samples (pus, aspirates, body fluids, lymph node [LN] tissue) were collected in special, plain, universal $30 \mathrm{ml}$ clear plastic container with cap (falcon tubes) under aseptic conditions. $5 \mathrm{ml}$ of samples were received to which buffer solution was added, then the mixture is loaded to cartridge which were processed by Xpert MTB/RIF assay (Cepheid-Sunnyvale-USA), as per the guidance document given by Central TB division, Government of India (RNTCP, 2013; RNTCP, 2012). The results can be read as MTB detected, MTB not detected, RIF resistance detected; RIF resistance not detected; RIF resistance indeterminate; or invalid/error with the help of positive beacons.

Inclusion criteria: Extra pulmonary samples (pus, aspirates, body fluids, LN tissue) from all the age group irrespective of gender.

Exclusion criteria: Blood samples, urine samples, sputum samples and any other EP sample contaminated with blood is not included in our study.

\section{Results}

Total of 281 extrapulmonary samples received during study period, 165(58.7\%) were males and 116(41.2\%) were females. Most of the cases were of age group 21 to 30 years. Out of 281 samples received, 73(25.97\%) were of FNAC, 115(40.92\%) Pleural fluid, 26(9.25\%) Ascitic fluid, 36(12.8\%) CSF, 22(7.82\%) Pus and 9(3.2\%) were aspirates (other body fluids like synovial fluid, vitreous humor etc) as shown in the table-1.

Among 281 samples, 67(23.8\%) were positive and 214(76.1\%) were negative for MTB as shown in figure-1. Out of 67 positives, 66(98.5\%) were MTB-positive/RIF resistance-negative and 1(1.49\%) was MTB- positive/RIF resistancepositive (in aspirate sample) as shown in the figure-2. Maximum number of MTB was detected in the age group of 20-30 years $(n=23,34.3 \%)$. Among 165 males and 116 females, MTB was detected in 44(26.6\%) and 23(19.8\%) respectively as shown in figure-3. Out of total 281 patients, 24(8.54\%) were HIV positive. Of these 24, only $8(33.3 \%)$ were found positive for MTB in different samples as shown in the table-2. Among 257 non-HIV patients, MTB was detected in $59(22.9 \%)$ as shown in table-3. Among different samples received, Pleural fluid was in maximum number $\mathrm{n}=115(40.9 \%)$. Maximum MTB positives were found in FNAC samples $n=35(52.2 \%)$ as shown in the figure-4.

Table-1: Different EPTB samples received and their percentages

\begin{tabular}{|c|c|c|}
\hline Specimen type & Frequency & Percentage \\
\hline FNAC & 73 & $25.97 \%$ \\
\hline Pleural fluid & 115 & $40.92 \%$ \\
\hline Ascitic fluid & 26 & $09.25 \%$ \\
\hline CSF & 36 & $12.81 \%$ \\
\hline Pus & 22 & $07.82 \%$ \\
\hline Aspirates & 09 & $03.20 \%$ \\
\hline
\end{tabular}


Original Research Article

Table-2: MTB detected in different samples received from HIV patients

\begin{tabular}{|c|c|c|}
\hline Samples & Total Positives & Percentage \\
\hline FNAC & 05 & $62.5 \%$ \\
\hline Pleural fluid & 01 & $12.5 \%$ \\
\hline Ascitic fluid & 00 & $00.0 \%$ \\
\hline CSF & 00 & $00.0 \%$ \\
\hline Pus & 02 & $25.0 \%$ \\
\hline Aspirates & 00 & $00.0 \%$ \\
\hline
\end{tabular}

Table- 3: MTB positives among HIV and Non-HIV patients.

\begin{tabular}{|c|c|c|c|}
\hline HIV status & Total & Mtb positive & Percentage \\
\hline Positive & 24 & 8 & $33.3 \%$ \\
\hline Negative & 257 & 59 & $22.9 \%$ \\
\hline
\end{tabular}

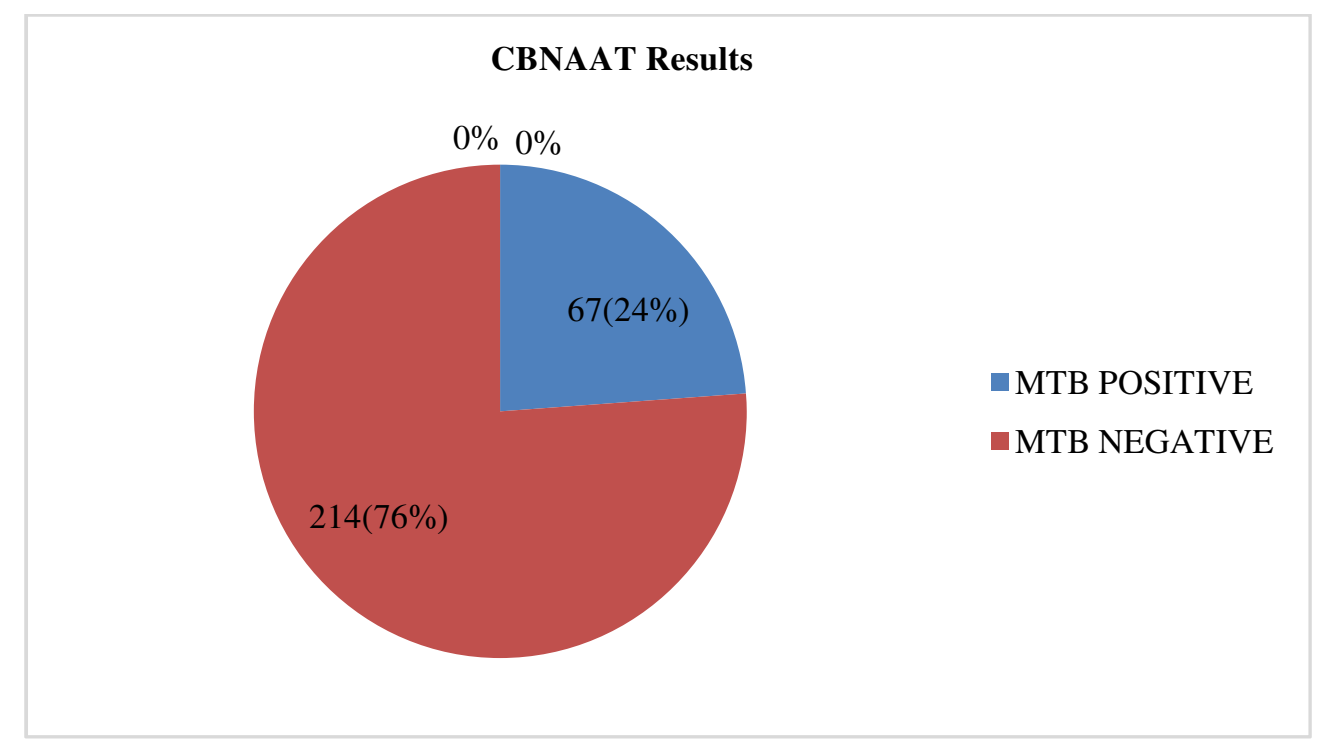

Figure 2: CBNAAT Results

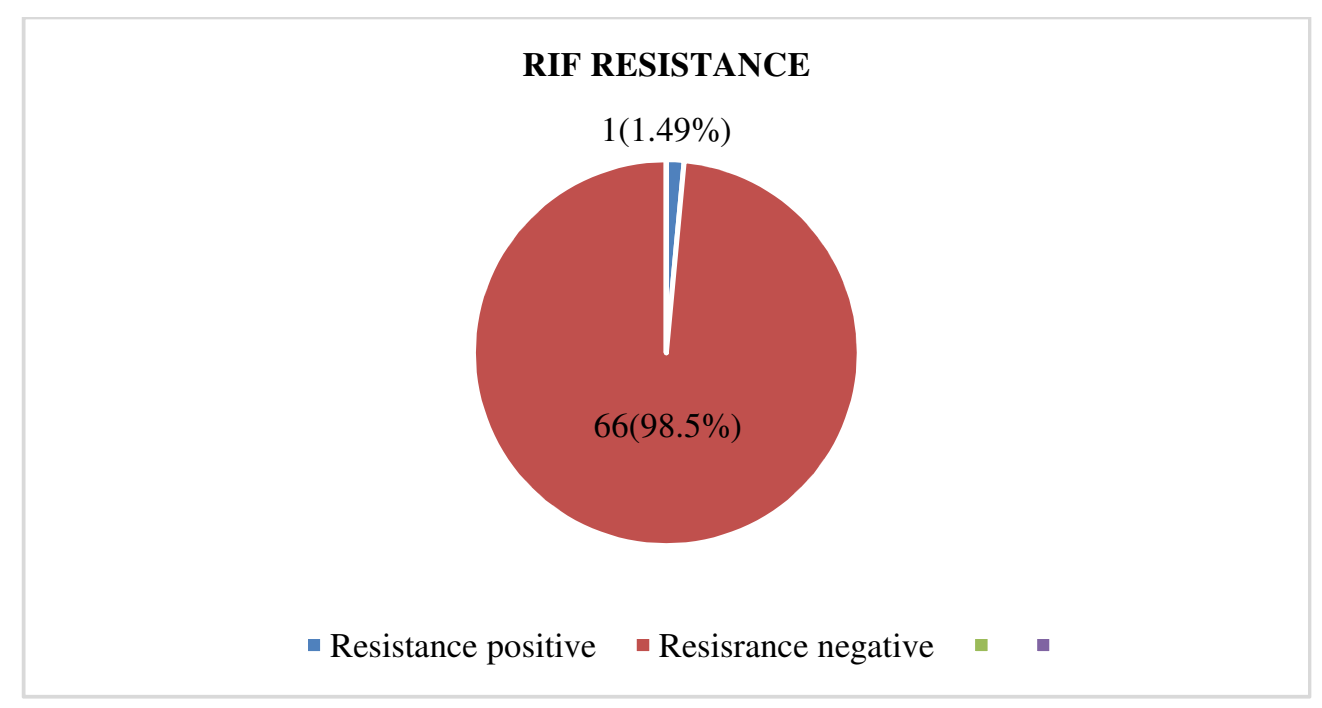

Figure- 3: RIF Resistance 


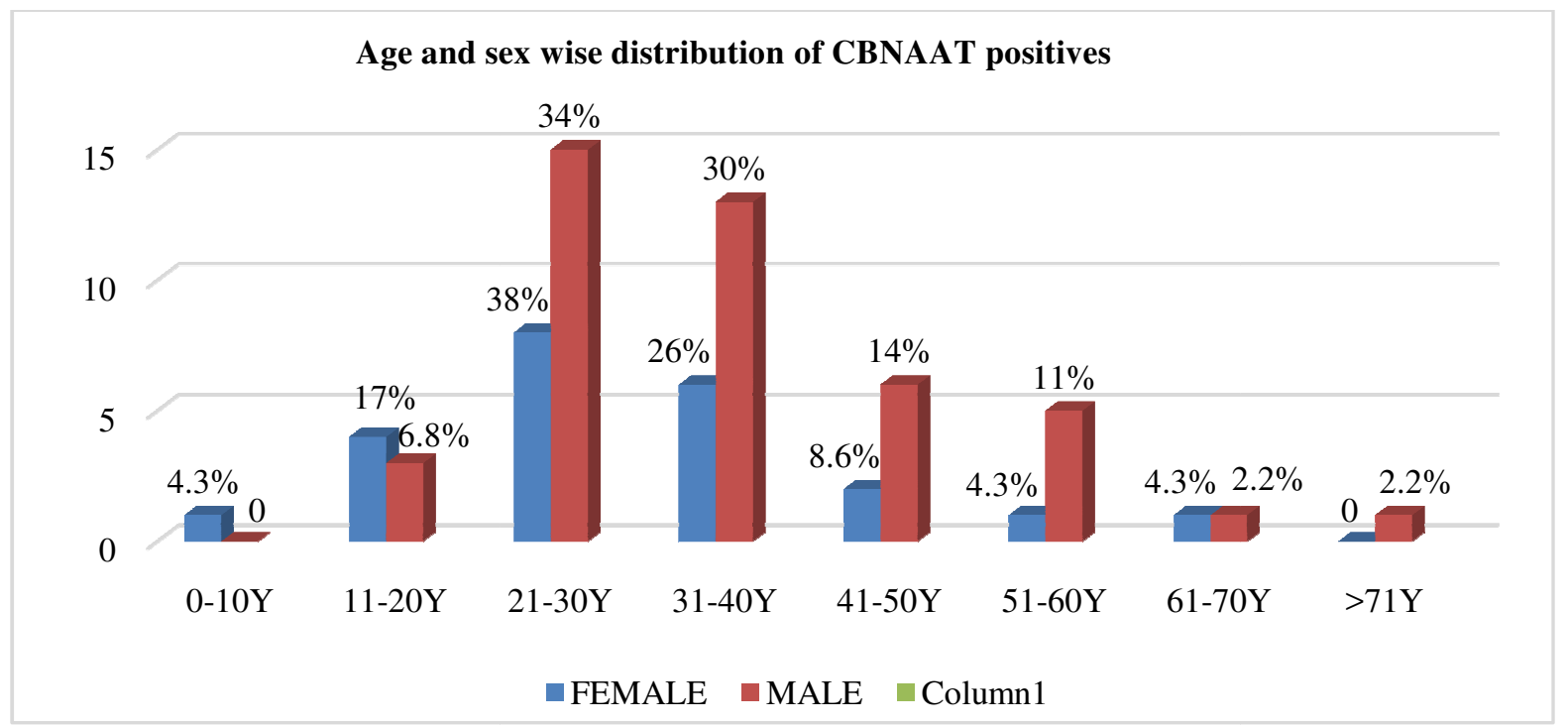

Figure 4: Age \& sex distribution of patients

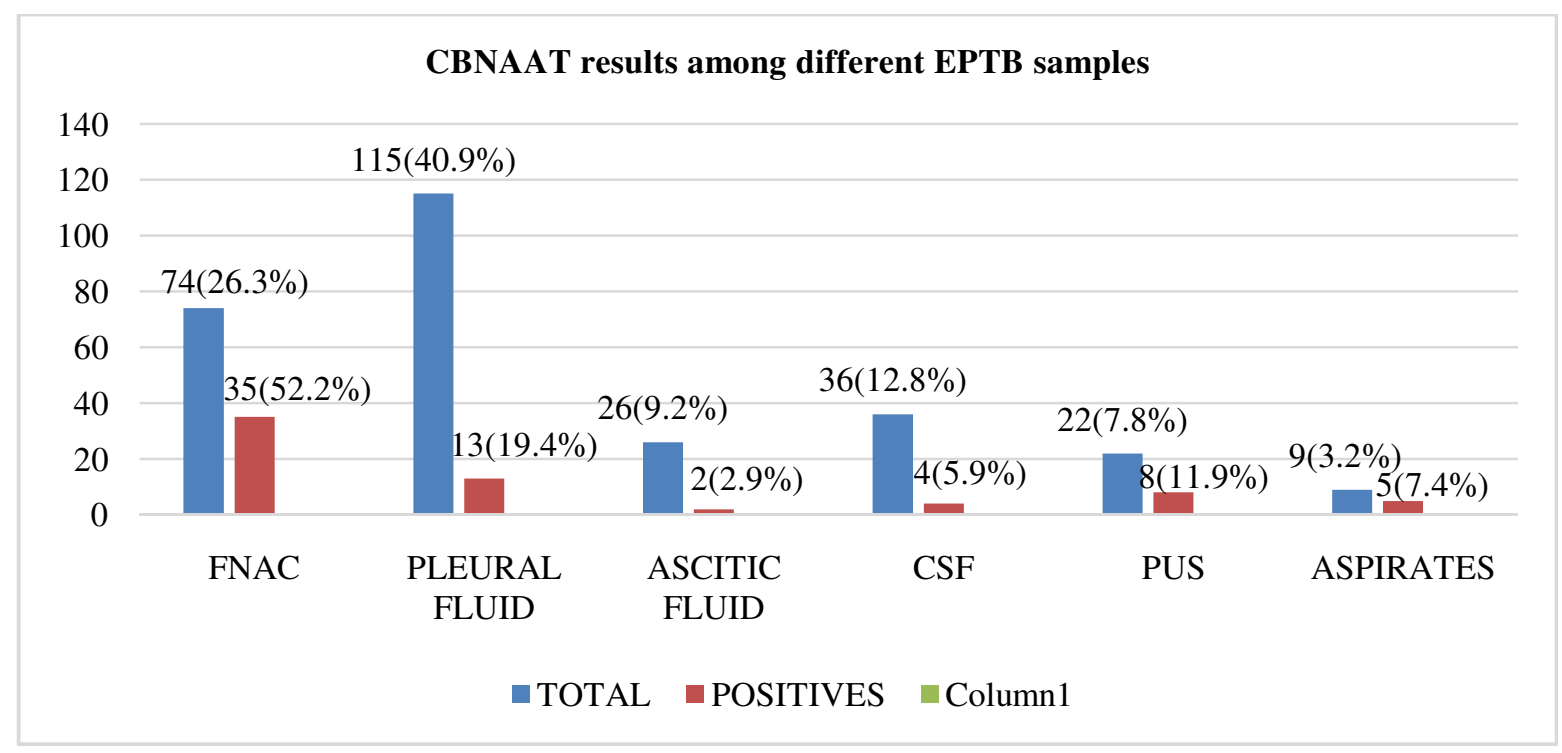

Figure 5: CBNAAT results among different EPTB Patients

\section{Discussion}

Extra pulmonary tuberculosis constitutes $20 \%$ of burden of TB globally. EPTB is a pauci-bacillary disease as number of bacteria are less. Conventional methods (histology and smear microscopy) are not diagnostic and diagnostic methods (culture methods) are time consuming. Therefore, a need for new and rapid diagnostic methods, nucleic acid amplification techniques like GeneXpert (CBNAAT) [7].

Numerous studies have assessed the yield of PCR techniques for the diagnosis of EPTB $[8,9,10]$. Sanjay $G$ $M$ et al., conducted a study on trends of EPTB and found higher detection of EPTB cases in younger age group [11]. Similar results were found in our study. Maximum cases were in the age group 21-30 years were
MTB was detected in $23(34.3 \%)$ cases. Our several findings are consistent with TB details of other studies like Balasubramanian et al., where males have higher MTB diagnostic rates compared to females [12]. A study conducted by Ullah I et al., on EPTB using CBNAAT detected MTB in 60 (35.7\%) samples, similar to our study where MTB detected in 67(23.8\%).

CBNAAT showed, $86 \%$ of Sensitivity, $88.4 \%$ of specificity and $71.7 \%$ of positive predictive value while negative predictive value was high i.e $95.1 \%$ in their study [13]. Scott L E et al., conducted a study on diagnostic accuracy of CBNAAT for EPTB specimens taking culture as reference, CBNAAT's overall sensitivity was low (59\%) while specificity was high 
(92\%) and Highest sensitivities was obtained for pus (91\%) followed by lymph node aspirates (80\%). Study also found that CBNAAT is not much affected by bacterial contamination reducing diagnostic delay compared to traditional methods like culture [14]. Sanjay $\mathrm{G}$ M et al found maximum cases of pleural effusion-133(49.81\%) with MTB detected in 13 $(9.77 \%)$. In our study maximum MTB cases were detected in FNAC samples-35 (47.9\%) of total 73 cases. Other samples processed were 115 cases of pleural fluid in which MTB detected in 13(11.3\%), 22 pus samples with MTB detected in 8(36.3\%), 26 ascitic fluid in which MTB detected in 2(7.69\%), 36 CSF samples with MTB detection in $4(11.1 \%)$ and 9 aspirates where 5 $(55.5 \%)$ were detected positive for MTB.

In a study done by Armand et al, among individual extrapulmonary samples, the sensitivity of CBNAAT was highest among lymph nodes (94.7\%). Inclusion of CBNAAT in the initial diagnosis of tubercular lymphadenopathy in addition to the FNAC would decrease the over diagnosis of tuberculosis and injudicious use of anti-tuberculosis treatment [15]. Similarly, in our study maximum MTB was detected in FNAC of LN samples.

Mittal M et al., on comparison of diagnostic yield of CBNAAT and ZN (Ziehl-Neelsen) staining from HIV and non-HIV patients with extra-pulmonary tuberculosis in their study showed of 81 extra pulmonary samples processed, 21(25.9\%) from HIV and 60(74\%) from non-HIV patients. Out of 21 HIV patients, $4(19.05 \%)$ and $9(42.85 \%)$ were positive for $\mathrm{ZN}$ stain and GeneXpert respectively. Among 60 non-HIV patients $7(11.66 \%)$ and $19(31.66 \%)$ were positive for ZN stain and GeneXpert respectively. Studies found that CBNAAT is more sensitive than $\mathrm{ZN}$ staining in EPTB while both HIV and non-HIV patients have a same yield [16]. In our study MTB was detected in 8 out of $24(33.3 \%)$ HIV patients while in non-HIV patients, MTB detected in 59 out of 257(22.9\%).

Molecular techniques have subsequently changed the field of tuberculosis diagnosis in both pulmonary and extra-pulmonary cases and have been proven to yield rapid results [17].

\section{Conclusion}

CBNAAT is a rapid test to confirm presence of MTB with simultaneous detection of rifampicin resistance in EPTB. Before the advent of CBNAAT, diagnostic test for all clinically suspected EPTB was staining method. Its specificity was very low because of which all
Original Research Article

clinically suspected EPTB cases use to receive empirical ATT which was a burden on both patient and health care system. Introduction of CBNAAT for EPTB, has an impact on early detection, treatment and outcome as most presumptive cases have a confirmed diagnosis. It has dramatically reduced the treatment abuse in suspected cases because of lack of confirmatory tests. This study also helped to know epidemiology of our locality.

Funding: Nil, Conflict of interest: None

Permission of IRB: Yes

\section{Reference}

1. World Health Organization. 2015. Global tuberculosis report, 2015. WHO/HTM/TB/2015.22. Geneva, Switzerland: WHO.

2. Tortoli E, Russo C, Piersimon C, Mazzola E, Monte P D, Pascarellae M, Borroni E, Mondo A, Piana F, Scarparo C, Coltella L, Lombardi G, Cirillo D M. Clinical validation of Xpert MTB/RIF for the diagnosis of extrapulmonary tuberculosis. Eur Respir J 2012;40 (2) : 442-447.doi: 10.1163/09031936.00176311.

3. Shetye s, Chheda P, Lad A, Matkar S, Yadav P. Utility of GeneXpert Mycobacterium tuberculosis/ Rifampin Assay for Extrapulmonary Tuberculosis Samples. MGM J Med Sci 2017;4(1):6-9.

4 Ramaswamy, S., and Musser, J.M. 1998. Molecular genetic basis of antimicrobial agent resistance in Mycobacterium tuberculosis. Tuber. Lung Dis 1998; 79 (1) : 3-29.doi: 10.1054/told.1998.0002.

5. Boehme CC, Nicol MP, Nabeta P, et al. Feasibility, diagnostic accuracy, and effectiveness of decentralised use of the Xpert MTB/ RIF test for diagnosis of tuberculosis and multidrug resistance: a multicentre implementation study. Lancet 2011;377(9776):14951505.doi: 10.1016/S0140-6736(11)60438-8.

6. Boehme CC, Nabeta P, Hillemann D, et al. Rapid molecular detection of tuberculosis and rifampin resistance. N Engl J Med 2010 Sep 9; 363: 1005-1015. doi: 10.1056/NEJMoa0907845.

7. Avashia S, Bansal D, Ahuja K, Agrawal V. Comparison of conventional methods with gene xpertmtb/rif assay for rapid detection of mycobacterium tuberculosis and rifampicin resistance in extrapulmonary samples. Int J of Med Res and Rev 2016 Feb 28; 4 (2):181-185.doi: 10.17511/ijmrr.2016.io2.010. 


\section{Original Research Article}

8. Cheng VCC, Yam WC, Hung IFN, Woo PCY, Lau SKP, Tang BSF, Yuen KY.Clinical evaluation of the polymerase chain reaction for the rapid diagnosis of tuberculosis. J Clin Pathol 2004; 57 (3):281-285.doi: http://dx.doi.org/10.1136/jcp.2003.012658.

9. Hasaneen N A, Zaki M E, Shalaby H M, El-Morsi A $\mathrm{S}$. Polymerase chain reaction of pleural biopsy is a rapid and sensitive method for the diagnosis of tuberculous pleural effusion. Chest 2003 Dec;124(6):2105-11.

10. Hofman V, Selva E, Landraud L, Sicard D, Venissac N, Castillo L, Kermarec A, Mouroux J, Dellamonica P, Hofman P. Value of PCR amplification from formalin-fixed paraffin-embedded tissues in the diagnosis of Mycobacterium tuberculosis infection. Ann Pathol 2003 Jun;23(3):206-215.

11. Gour Sanjay M, Munje Radha P, Neenu P Babu, Muley Sushant, Atram Jitesh. Genotypic diagnosis of extra pulmonary tuberculosis - CBNAAT a novel tool. MedPulse International Journal of Medicine. 2017 Nov 2 ; 4(2): 79-82. doi: https://doi.org/10.26611/1021425.

12. Balasubramanian, R., R. Garg, T. Santha, P.G. Gopi, R. Subramani, Chandrasekaran, V. et al. 2004. Gender disparities in tuberculosis: report from a rural DOTS programme in south India. Int J Tuberc Lung Dis 2004 Mar; 8(3): 323-32.

13. Ullah I, Javaid A, Masud H, Ali M, Basit A, Ahmad W, Younis F, Yasmin R, Khan A, Jabbar A, Husain M.
Rapid detection of Mycobacterium tuberculosis and rifampicin resistance in extrapulmonary tuberculosis and sputum smear-negative pulmonary suspects using Xpert MTB/RIF. J Med Microbiol 2017 Apr 28; 66(4): 412-8.doi: 10.1099/jmm.0.00049.

14. Scott LE, Beylis N, Nicol M, Nkuna G, Molapo S, Berrie L, Duse A, Stevens WS. Diagnostic accuracy of Xpert MTB/RIF for extrapulmonary tuberculosis specimens: establishing a laboratory testing algorithm for South Africa. J Clin Microbiol 2014 Jun 1;52(6): 1818-23.doi: 10.1128/JCM.03553-13.

15. Armand S, Vanhuls P, Delcroix G, Courcol R, Lemaitre N. Comparison of the Xpert MTB/RIF test with an IS6110-TaqMan real-time PCR assay for direct detection of Mycobacterium tuberculosis in respirstory and nonrespiratory specimens. J Clin Microbiol. 2011; 49 (5): 1772-1776. doi: 10.1128/JCM.02157-10.

16. Mittal M, Kumar R. Comparison of diagnostic yield of GeneXpert MTB/RIF assay and ZN (Ziehl-Neelsen) staining in serosal fluids from HIV and non-HIV patients with extra-pulmonary tuberculosis. Int J Res Med Sci 2017Jul;5(7):2952-2955.doi: http:/dx.doi. org/10.18203/2320-6012.ijmrs20172967.

17. Boehme C C,Nabeta P,Hillemann D, Nicol M P. Rapid molecular detection of tuberculosis and rifampin resistance. N Engl J Med 2010 Sep 9;363:1005-1015. doi: 10.1056/NEJMoa0907847.

\section{How to cite this article?}

Yadhav K, Veena M. Role of GeneXpert in Rapid Molecular Detection of Extrapulmonary Tuberculosis in Tertiary Care Hospital. Int J Med Res Rev 2018;6(05):271-276.doi:10.17511/ijmrr. 2018.i05.06. 